\title{
Mixing and Internal Waves in a Small Stratified Indian Lake: Subhas Sarobar
}

\author{
N.R. Samal, Klaus D. Jöhnk ${ }^{1}$, Frank Peeters, \\ Erich Bäuerle ${ }^{2}$ and Asis Mazumdar ${ }^{3}$
}

Limnologisches Institüt, University of Konstanz, Germany

Nihar.Samal@uni-konstanz.de,niharwre@sify.com

${ }^{1}$ Aquatic Microbiology/Institute for Biodiversity and Ecosystem Dynamics

University of Amsterdam, The Netherlands

${ }^{2}$ Wasseransichten, Germany

${ }^{3}$ School of Water Resources Engineering, Jadavpur University, Kolkata, India

\section{INTRODUC'TION}

The formation and break-up or erosion of stratification is a major process in all natural and man-made lakes, which controls to a large extent the functioning of their ecosystems. The key challenge here is the specific role of shear generated and convective turbulence in the formation/destruction of stratification and their interactions with internal waves. Three aspects of hydrodynamics, i.e. the dynamical state, the physical mechanism and the energy level are very important elements in understanding problems of turbulent mixing and the formation of vertical thermal stratification. The result of the stratification is the formation of the seasonal thermocline.

Mixing dynamics is of great importance for the management of water quality because it allows for water pollution prediction in relation to thermal stratification. In particular vertical mixing is an important aspect with respect to the exchange processes of heat and dissolved substances between the different vertical layers of the lake. It is controlled by atmospheric forcing at the water surface and the resultant advective and oscillatory motions in the water column. Generally speaking, the intensity of vertical mixing decreases with depth. Traditionally, mixing is expressed in terms of a turbulent mixing coefficient in analogy to the molecular diffusion coefficient. The turbulent coefficients are a measure of the intensity of the process and may subsequently be used in calculations related to water quality and heat transport. Mixing can be assessed either directly from microstructure measurements of 
the turbulence levels or indirectly by measuring temporal changes of the spatial gradients of some particular properties such as temperature.

In the present investigation, the seasonal cycle of thermal stratification has been simulated for a period of more than five years based on hourly meteorological input with a one-dimensional (vertical) turbulence model (LAKEoneD). Using these temperature-depth (TD) profiles, the annual cycle of phase velocities of long internal waves is calculated depending on the density difference in the stratified lake. Based on the knowledge of the actual value of the phase velocity the spectra of free internal oscillation can be calculated. That is done for the periods of significant stratification (April to November) by using a horizontally two-dimensional eigen-frequency model. From the calculations it becomes clear that the first order baroclinic free oscillations have periods significantly smaller than diurnal or semi-diurnal values. Thus, resonance with meteorological forcing on that time scale can be ruled out. Changing spatial patterns of observed temperature distributions indicate that the small shallow lake in the tropics exhibits differential mixing which may cause horizontal exchange flows between different stations.

\section{LAKE CHARACTERISTICS AND DATA}

Study site: Subhas Sarobar (Latitude $22^{\circ} 34^{\prime}-22^{\circ} 34^{\prime} 30^{\prime \prime} \mathrm{N}$ and Longitude $88^{\circ} 24^{\prime}-88^{\circ} 24^{\prime} 30^{\prime \prime} \mathrm{E}$ ), under the administrative control of Kolkata Improvement Trust (KIT), represents the lung of East Kolkata with massive environmental fillip. The lake ecosystem is playing a key role in maintaining the oxygen balance and is also being used for sports, recreational and cultural activities. The vast water body and its two islands, one small and the other big, constitute an excellent habitat for diverse species of life and also have got potential for attracting the tourists. The water area of Subhas Sarobar (Fig. 1) is about 16.0 ha. The maximum length and width of the lake are

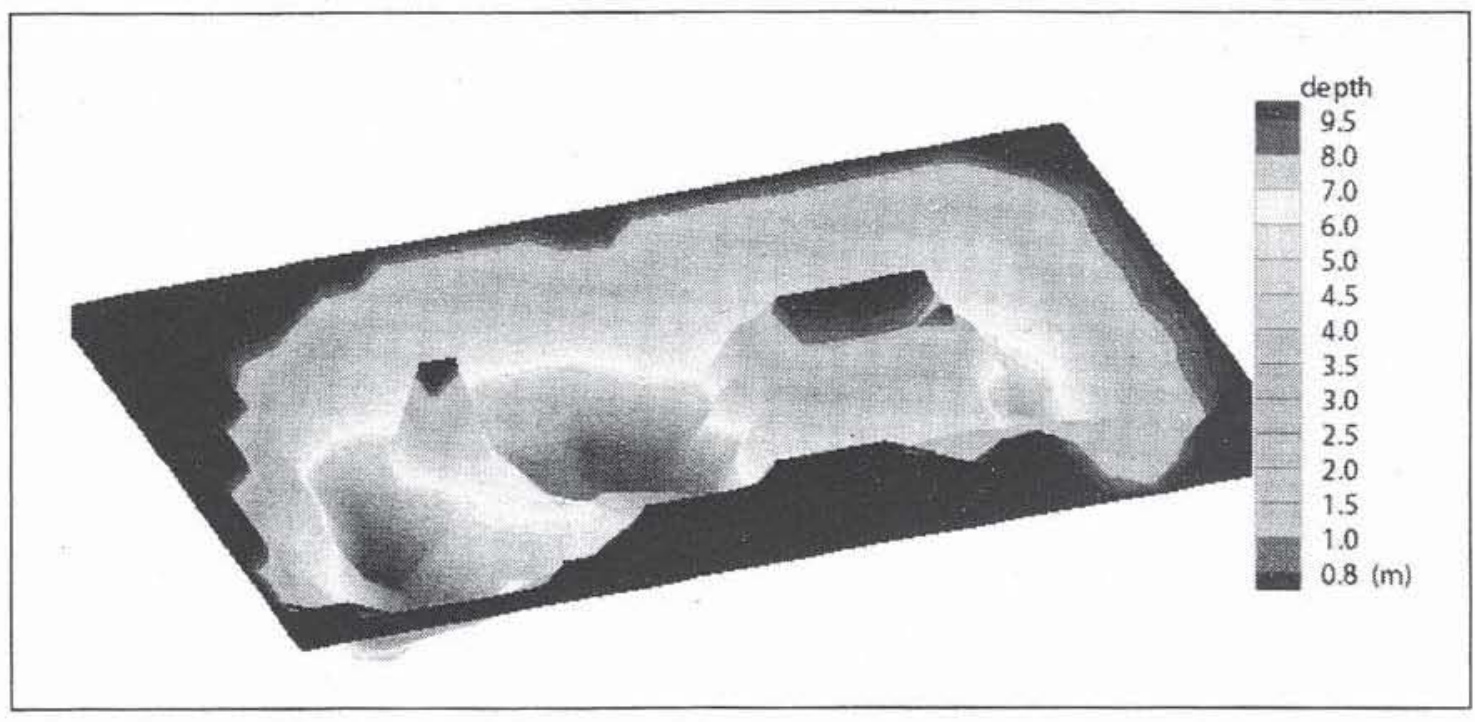

Figure 1: Three-dimensional view of the topography of lake, Subhas Sarobar, Kolkata, India. 
$617 \mathrm{~m}$ and $352 \mathrm{~m}$ respectively while the maximum depth and the mean depth of the lake are $10 \mathrm{~m}$ and $4.8 \mathrm{~m}$, respectively. The waterbody is given on rent to the Department of Fisheries of Government of West Bengal state. The open space in the lake is an oasis in the space-limited city. Recently, the pressure of human activities on the Subhas Sarobar has increased manifolds. Over three thousand of people per day are using it for washing of clothes and utensils and for bathing.

Lake temperature data: The observation sites are selected considering the maximum water depth along the mid-reach (Station I, II and III) which are also least influenced by human intervention, to study the thermal stratífication. The measurement of temperature profiles in this lake have been started during the month of May, 2003 using a WTW temperature/oxygen meter (Germany) along the depth at an interval of $0.5 \mathrm{~m}$ below the water surface, thus providing detailed information on the temperatures prevailing in all depth regions of the lake. All temperatures are considered to be accurate to within $\pm 0.1^{\circ} \mathrm{C}$. Also some temperature profiles during the year 1999 are recorded by the Institute of Wetland Management and Ecological Design, Kolkata (Saha, 2000).

Meteorological data: The daily meteorological data, i.e. maximum and minimum air temperature, maximum and minimum relative humidity, mean wind speed and mean cloud cover were supplied by the Dumdum meteorological station (Kolkata), located within $9 \mathrm{~km}$ of the northern lake shore and also from the internet site Weather Underground (Pawson, 2004). In order to take adequate account of the diurnal cycle in meteorological forcing, which may be important for the long-term development of the thermal structure of the lake, the meteorological data are interpolated at hourly intervals. It is demonstrated that the numerical lake model is able to simulate the main features of the thermal structure of a lake without the luxury of accurate, high-resolution input data. The empirical equations for the interpolation of the meteorological data into hourly values are described somewhere else (Samal, 2004). The hourly values of the global irradiance are calculated depending on the course of the sun from the geographical position, cloud cover, $\mathrm{C}$, and wind speed, $\mathrm{U}$.

Light extinction coefficient: As the solar radiation is absorbed within the water column rather than at the air-water interface, the vertical distribution of heat depends not only on vertical mixing processes, but also on light extinction coefficient. The extinction coefficient for clear water is determined by the empirical formula given in Joehnk and Umlauf (2001)

$$
K_{\mathrm{cw}}=1.7 / Z_{\text {secchi }}
$$

From the maximum measured Secchi-depth of $Z_{\text {secchi }}=1.7 \mathrm{~m}$ during our observation period, we infer a clearwater extinction of $K_{\mathrm{cw}}=1.00 \mathrm{~m}^{-1}$. 


\section{SIMULATION MODELS}

\subsection{Description of the Turbulence Model}

The two-layer model: Turbulent kinetic energy in a lake is generated by the wind shear stress that acts at the lake surface due to meteorological activity above the lake. This causes a vertical mixing within the water column and therefore influences not only the temperature distribution but also the average residence time of the planktonic organisms in the euphotic zone and thus determines their survival conditions. As compared to the vertical variation of the dominant processes the horizontal water motion can be ignored if longterm processes and global dynamics are examined. In this case it suffices to establish a model for an arbitary offshore water column of specified depth, valid for an arbitrary lake.

Every temperature and density profile corresponds to a particular value of potential energy of the water column, which follows from the centre of gravity of this water column. The considered physical processes in a lake essentially amount to a mutual exchange between this potential energy and the turbulent kinetic energy (TKE) caused by wind shear stress and surface cooling. The centre of gravity of a stable density profile always lies below the centre of gravity of a homogeneous profile. Thus, kinetic energy must be expended to homogenize the stratified column or part of it, and conversely, an unstable water column neutralises itself automatically by transforming potential energy into turbulent kinetic energy (which in turn may homogenize a sublaying stable profile so as to re-transform itself via convection again into potential energy).

Heat flow over the surface of the domain and volume of production of heat by solar irradiance change, in general, both the location of the centre of gravity of a water column as well as its heat content. The mechanical wind energy induced into the lake by turbulence activity and the mixing of the water by convection, however, only change the potential energy, maintaining the heat content of the water column.

The vertical transport of the TKE that is generated by the surface wind shear or the cooling of surface water is substantially simplified by the twolayer assumption: according to this simplification the entire TKE is at all times uniformly distributed in the mixed layer, whilst the underlying water remains at rest. By this drastic assumption the TKE profile, that decreases rather exponentially, is averaged in the upper and neglected in the lower layer, so that the model becomes 'zero-dimensional' in respect to the TKE distribution. This two layered model was theoretically proposed and experimentally verified by Kraus and Turner (1967) and was further developed, e.g., by Niiler (1977) to simulate the thermal stratification in the ocean. The actual interface between the two layers, the diurnal turbocline, may be identified with the largest vertical gradient of the TKE in real lakes. 
Its location does not, in general, agree with the location of the thermocline. However, the seasonal turbocline that corresponds to the daily maxima of the diurnal turbocline can be identified with the depth of the epilimnion and thus with the seasonal thermocline.

The external driving processes influence the content of the TKE in the mixed layer as follows:

(i) The wind input generates TKE by establishing a vertical shear and producing breaking surface waves.

(ii) Heat flow over the free surface into the atmosphere increases the density and thus lowers the buoyancy of the uppermost layers (as long as the temperature stays above the density maximum). The heavy water sinks and mixes itself turbulently with the underlying layers until homogenized conditions are re-established. The TKE of the mixed layers rises.

(iii) Conversely, heat flow from the atmosphere into the lake raises the buoyancy of the uppermost layers. TKE produced by the wind shear must be expended to homogenize the mixed layer by mixing the stable upper layer against gravity into the lower and colder layers.

(iv) The non-uniform heating of the mixed layer by solar radiation stabilizes the water column in a similar way as a heat flow across the free surface, since the absorbed radiative heat is exponentially attenuated with depth. This process also absorbs TKE.

(v) When the mixed layer is deepened, the cold water from below the turbocline must be mixed with the water above, a process that also consume TKE.

On the basis of the two-layer assumption, a positive TKE balance of the processes (i) - (iv) is instantly and completely compensated by lowering the turbocline into deeper layers (entrainment) and thus consuming the abundant TKE via processes (v). In the case of a negative balance, the turbocline is elevated (detrainment) to reduce the depth to which warm surface water has to be mixed down.

In the one-dimensional turbulence model used here, the restriction to such a simplified two-layer system is lifted. Driven by hourly meteorological fields it calculates temperature, turbulent kinetic energy and turbulent dissipation rates on a depth grid with $d z=0.25 \mathrm{~m}$ and on time steps of four minutes.

\subsection{MODEL EQUATIONS}

The evolution of all variables is determined by the one-dimensional vertical diffusion equation with time and space dependent vertical diffusion coefficients which are discussed in great detail in Jöhnk and Umlauf (2001) and Hutter and Jöhnk (2004). The equations for temperature, turbulent kinetic energy 
and its dissipation together with the balance equations for momentum in horizontal direction are solved numerically using a implicit time integration method.

\subsubsection{Internal Oscillations}

During the periods of stratification, the isotherms nearly never stay at horizontal levels (as in the case of the undisturbed equilibrium) but exhibit periodic fluctuations both in time and space (internal waves and internal oscillations). In a small lake the internal waves are reflected multifold by the nearly omnipresent lateral boundaries, resulting in the quasi-standing patterns of internal oscillations. Although in tropical lakes the temperature differences between epilimnion and hypolimnion are only a few degrees, due to the high absolute values the vertical density gradients are rather large and the stability of the water column is relatively strong. Consequently, the phase velocities of long internal waves are comparable with the values, which are known from the deep lakes of the temperate zones.

Due to the short cross-lake distances it is clear that the periods of free internal oscillations in a small lake should not be larger than a few hours.

We make profit of the fact that under the assumption of flat bottom the spatial dependency of the variables can be separated into a purely vertical and a purely horizontal dependency, respectively (Charney, 1955). The differential equation for the vertical dependency is given by

$$
\frac{d^{2} \hat{Z}}{d z^{2}}+\frac{N^{2}(z)}{g h_{\mathrm{n}}} \hat{Z}=0,
$$

which together with the boundary conditions at the surface (rigid lid assumption)

and at the bottom

$$
\hat{Z}=0 \text { at } z=0
$$

$$
\hat{Z}=0 \text { at } z=H
$$

form a classical Sturm-Liouville problem for the eigenvalue $g h_{n}$, which in the present case is the square of the phase velocity of long internal waves. $N(z)$ is the Brunt-Väisälä frequency defined by

$$
N^{2}(z)=\frac{g}{\rho} \frac{d \rho}{d z},
$$

where $\rho(z)$ is the vertical density distribution, which in fresh water lakes is mainly determined by the temperature.

Once $c_{\mathrm{n}}$ is calculated, the system of differential equations governing the horizontal dependency is given by

$$
i \omega U+f V+g h_{\mathrm{n}} \frac{\partial Z}{\partial x}=0,
$$




$$
\begin{aligned}
i \omega V-f U+g h_{\mathrm{n}} \frac{\partial Z}{\partial y} & =0, \\
i \omega Z+\frac{\partial U}{\partial x}+\frac{\partial V}{\partial y} & =0 .
\end{aligned}
$$

Here, $f$ is the Coriolis parameter $\left(f=0.56 \times 10^{-4} \mathrm{~s}^{-1}\right.$ for Subhas Sarobar $)$, $U, V, Z$ are the (complex) amplitudes of the components of the horizontal velocity and the vertical displacement of the isothermes, respectively. By solving the systems of equations numerically for the eigenvalues $(\omega)$, we get a set of eigenfrequencies, which may be ordered in a chronological way $\left(\omega_{1}<\omega_{2}<\omega_{3}<\ldots \ldots.\right) . \omega_{1}$ is the eigen frequency of the so-called fundamental horizontal mode of oscillation.

The eigen frequencies of the free internal oscillations in the stratified lake are calculated with a proved numerical finite difference model (Bäuerle, 1985).

\section{RESULTS AND DISCUSSION}

\subsection{Simulation of Temperature Stratification}

The temperature stratification of the lake, Subhas Sarobar has been simulated for the years 1999 to May, 2004. The model is calibrated by comparing the results of simulations with measurements. The model run is initialised by applying the prescribed atmospheric forcing to a water body at uniform temperature, close to the average air temperature. The total short wave irradiance is derived semi-empirically as described somewhere else (Samal, 2004), all other meteorological parameters are taken from the measured values of a nearby weather station. The results for the year of comparison of simulation with measured data are displayed in the panels of Fig. 2.

The figures show an appreciable good agreement to the measured data. A simple quantification of the goodness of simulation results for individual profiles at different stations within the lake-although the simulation is run only with respect to the deepest point-is made by calculating the mean squared error. The low temperature as observed in the first profile of the measured data as compared to profiles of other date between different stations might be due to differential heating. or differential mixing, respectively.

In Fig. 3 the phase velocity of the first baroclinic mode is shown for five years of numerical calculations. Herewith, it is possible to estimate the spectra of internal oscillations.

\subsection{INTERNAL OSCILLATION}

For the three lowest modes of internal oscillations of the equivalence depth model the dependency of the eigenfrequencies on the phase velocity of long 


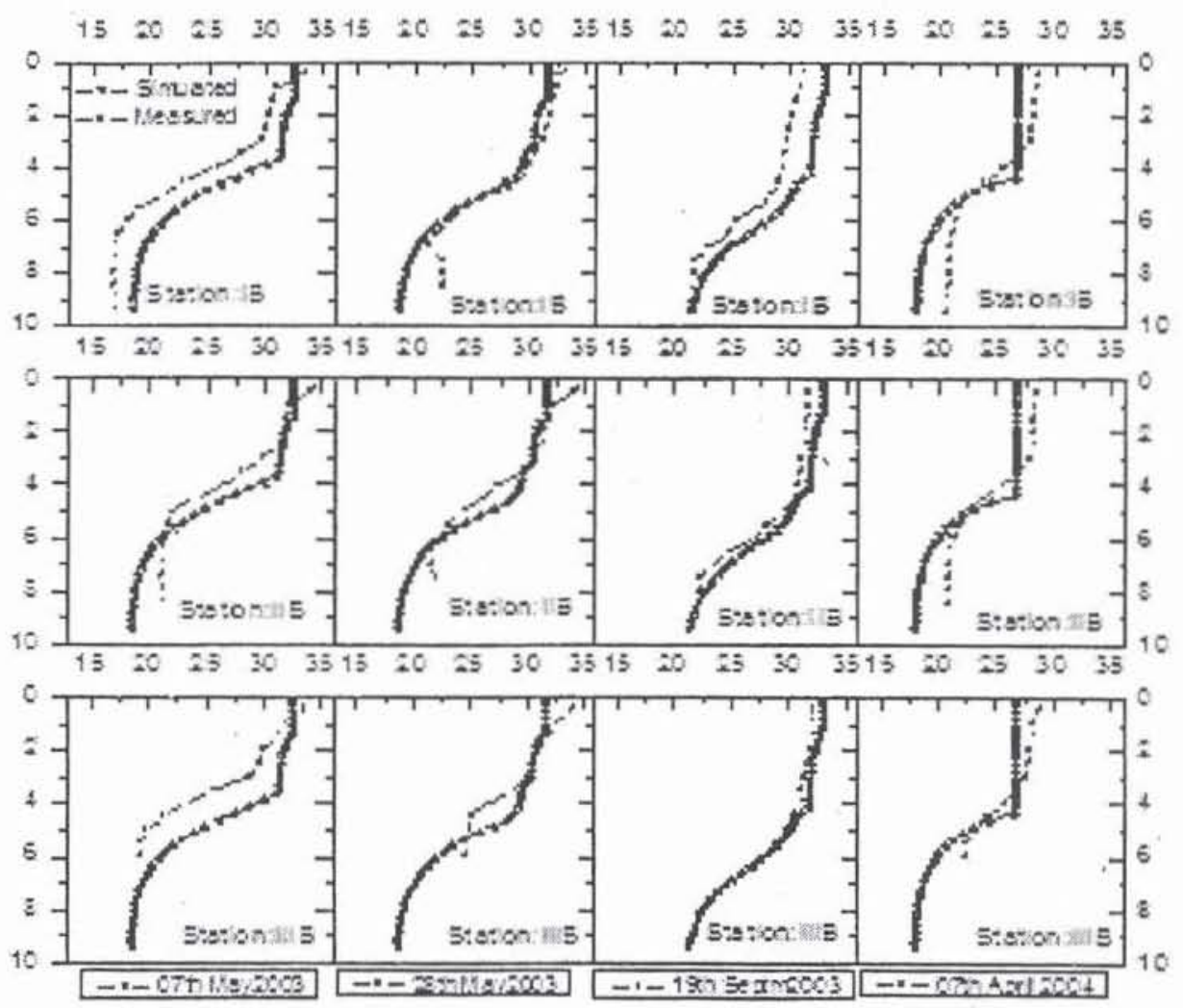

Figure 2: Measured and simulated temperature profile of lake Subhas Sarobar.

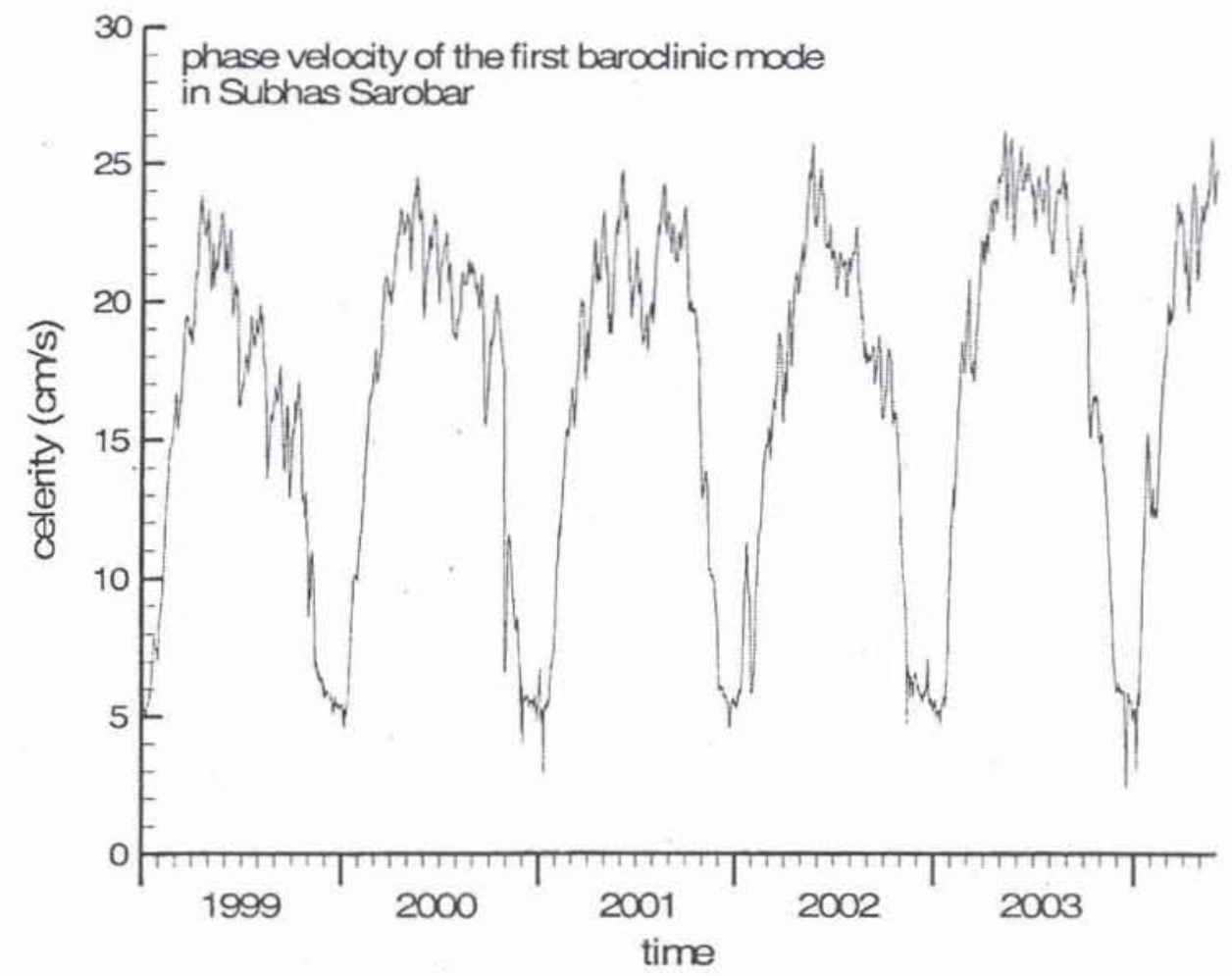

Figure 3: The development of the phase velocity (celerity) of the first vertical mode of long internal waves in Subhas Sarobar from 01.01.1999 to 31.05 .2004 . The depth of the basin is assumed to be $9.25 \mathrm{~m}$. Stratification is taken from a one-dimensional turbulence model driven by the observed meteorological forces (profiles at 12:00 hours are taken). 
internal waves is shown in Fig. 4. The curves follow approximately the formula

$$
T_{\mathrm{n}}=\frac{2 L^{(\mathrm{n})}}{c} \text { or } \omega_{\mathrm{n}}=\frac{\pi c}{L^{(\mathrm{n})}},
$$

where $L^{(\mathrm{n})}$ is a (mode-dependent) length scale, $c$ is the phase velocity of long internal waves (first baroclinic order). The value of $25.0 \mathrm{~cm} / \mathrm{s}$ corresponds to very strong stratification as observed in summer (Fig. 3). The value of $4.43 \mathrm{~cm} / \mathrm{s}$ could happen very early in spring or very late in autumn.

We get the length scales $L^{(\mathrm{n})}$ for Subhas Sarobar. by calculating the eigenfrequencies of the model lake for a certain stratification which is represented by a specific value of the phase velocity $c_{\mathrm{r}}$ as a reference (Table 1). Obviously, $L^{(1)}$ and $L^{(2)}$ correspond to the mean length and the mean width of the lake, respectively.

Table 1: The eigenfrequencies and length scales of the four lowest internal seiches in Subhas Sarobar without Earth's rotation.

The values of the second column refer to $c_{\mathrm{r}}=4.43 \mathrm{~cm} / \mathrm{s}$.

\begin{tabular}{cccc}
\hline Mode & $\omega_{n}\left(\times 10^{-4} s^{-1}\right)$ & $\omega_{n} / \omega_{l}$ & $I^{(n)}(m)$ \\
\hline 1 & 2.25 & 1.000 & 618 \\
2 & 3.62 & 1.627 & 380 \\
3 & 4.34 & 1.929 & 320 \\
4 & 5.04 & 2.240 & 276 \\
\hline
\end{tabular}

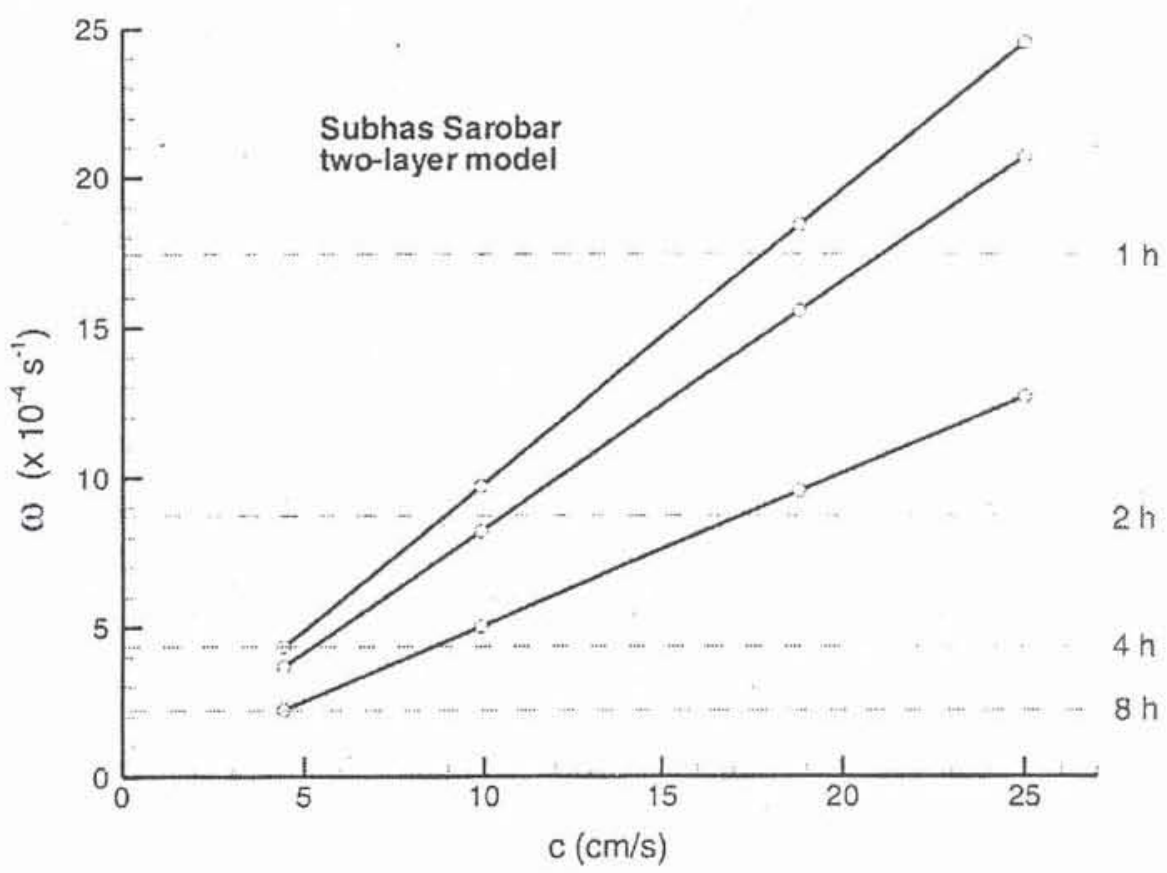

Figure 4: The dependency of the three lowest modes of internal free oscillations in an equivalence-depth model of Subhas Sarobar on the phase velocity of long internal waves. The range of $c$ corresponds to the values of Fig. 3 . 


\section{CONCLUSIONS}

The application of a vertical, one-dimensional turbulence model to the tropical lake Subhas Sarobar showed good agreements with temperature measurements at that lake. The calibrated model can now be used to simulate temperature profiles and mixing intensity depending on meteorological forcing. Several applications are possible. Firstly the model can be used to hindcast temperature profiles to fill in measurement gaps in previous years. Secondly it can be used to predict the temperature development in the lake based on actual weather conditions. And lastly it is possible to forecast lake temperature and mixing conditions in the lake under future climate change scenarios. The theoretical findings concerning internal oscillations need observational confirmation. However, based on the results of the numerical calculations it is clear that the main energy of the internal oscillations is at periods of a few hours. With a minimum of temperature sensors it would be possible to throw light on that fascinating subject.

\section{REFERENCES}

Bäuerle, E., 1985. Internal free oscillations in the Lake of Geneva. Annales Geophysicae, 3: 199-206.

Charney, J.G., 1955. Generation of oceanic currents by winds. J. Mar: Res., 14: 477498.

Hutter, K. and Jöhnk, K.D., 2004. Continuum methods of physical modelling Continuum mechanics, dimensional analysis, turbulence. Springer-Verlag, Berlin Heidelberg, pp. 635.

Imberger, J., 1998. Flux paths in a stratified lake: Review, Physical processes in lakes and oceans, Coastal and Estuarine studies. American Geophysical Union, 54: 9-10.

Jöhnk, K.D., 2005. Heat Balance of Open Water Bodies. In: Lehr, J.H. (ed.), The encyclopedia of Water. Wiley \& Sons (in press).

Jöhnk, K.D. and Umlauf, L., 2001. Modelling the metalimentic oxygen minimum in a medium sized alpine lake. Ecol. Model., 136: 67-80.

Kraus, E.B. and Turner, J.S., 1967. A one-dimensional model of the seasonal thermocline - II. The general theory and its consequences. Tellus, 19: 98-105.

Pawson, L., 2004. Weather Underground, http://www.wunderground.com

Niiler, P.P., 1977. One-dimensional models of the seasonal thermocline. In: Goldberg, E.D. (Ed.), The Sea, pp. 97-115.

Saha, T.K., 2000. Final report on "Monitoring of environmental status of Subhas Sarobar, Calcutta and preparation of management action plan" (01.08.1998 31.01.2000), Institute of Wetland Management and Ecological Design, Salt Lake, Calcutta - 700091.

Samal, N.R., Roy, D., Mazumdar, A. and Bose, B., 2004. "Sustenance of aquatic life in a thermally stratified tropical shallow lake", International Conference on Environment and Development organised by Institute of Social Science and West Bengal State of Technical Education, DST, Govt. of West Bengal, India.

Samal, N.R., 2004. Study of morphometry and hydrothermal analysis of a tropical shallow Indian lake, report submitted to DAAD (A/04/06852), Bonn, Germany. 